\title{
An Approach to a Rare Case of Collision Tumor of Follicular and Papillary Carcinomas of the Thyroid
}

\author{
Rahul S Gosavi ${ }^{1}$, Akash D Gupta ${ }^{2}$, Shishir D Gosavi ${ }^{3}$, Milind H Patwardhan ${ }^{4}$
}

\begin{abstract}
Aim and objective: The main aim of reporting this case is to make the clinician aware of a rare collision tumor of the thyroid gland and a systematic approach to such cases.

Background: Collision tumors, though reported frequently in other organs, are an uncommon pathology in the thyroid gland and present as a diagnostic and treatment challenge.

Case description: This is a case of follicular and papillary carcinomas occurring simultaneously in different lobes of the thyroid gland as a collision tumor. This is an extremely rare entity, with only three cases found to be reported on a detailed search through the literature.

Clinical significance: Management of such dual pathology tumors is complex and should be patient-specific and directed toward more aggressive tumors; however, such clinical scenario lacks standardized diagnosis and management protocol due to lack of comprehensive knowledge regarding the behavior of such collision tumors. This case report is therefore an attempt to guide the clinician in the management of such cases. Keywords: Collision tumor, Follicular carcinoma thyroid, Papillary carcinoma thyroid, Thyroid malignancy.

Otorhinolaryngology Clinics: An International Journal (2020): 10.5005/jp-journals-10003-1353
\end{abstract}

\section{INTRODUCTION}

Collision tumors are defined as geographically coexistent but histologically distinct and morphologically independent

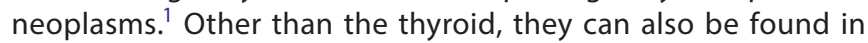
various other organs, such as, the ovaries, colon, lung, stomach, and kidneys. ${ }^{2}$ Literature reviews of collision tumor of thyroid have shown various combinations of carcinomas occurring simultaneously, and the medullary-papillary carcinoma variant was found to be the most common. ${ }^{3}$ However, the combination of follicular carcinoma and papillary carcinoma occurring as a collision tumor was rare and only three cases were found to be reported till date. ${ }^{4-6}$

\section{Case Description}

A 32-year-old male presented to our outpatient department with a right-sided anterior neck swelling of 18 months duration with no other associated symptoms. On examination, he was found to have a solitary nodule in the right lobe of thyroid measuring $6 \mathrm{~cm} \times 4 \mathrm{~cm}$, firm in consistency with mild deviation of the trachea to the left side and no palpable lymph nodes. Rigid videolaryngoscopy revealed normal vocal cord movements.

Thyroid function tests were normal. Ultrasonography of the neck showed a $6 \mathrm{~cm} \times 3.6 \mathrm{~cm} \times 3.5 \mathrm{~cm}$ iso- to hypo-echoic nodule in the right lobe of thyroid. The left lobe of thyroid and isthmus were reported as normal. Fine needle aspiration cytology (FNAC) from the swelling was reported as a follicular lesion of undetermined significance (Bethesda grade III) of the thyroid gland. Hence, a right hemithyroidectomy was planned and performed accordingly. Postoperative hospital stay of the patient was uneventful.

Histopathological examination of the resected specimen showed it to be having a solitary nodule of size $4.5 \mathrm{~cm} \times 2.5 \mathrm{~cm} \times 2 \mathrm{~cm}$ with a completely encapsulated neoplasm, arranged in follicular

\footnotetext{
${ }^{1-3}$ Department of ENT, Sanjeevan Medical Foundation ENT PG Training Institute, Miraj, Maharashtra, India

${ }^{4}$ Department of Endocrinology, Diabetic and Endocrine Research Centre, Vantmure Corner, Miraj, Maharashtra, India

Corresponding Author: Rahul S Gosavi, Department of ENT, Sanjeevan Medical Foundation ENT PG Training Institute, Miraj, Maharashtra, India, Phone: +91 9923203801, e-mail: drrahulgosavi88@gmail.com

How to cite this article: Gosavi RS, Gupta AD, Gosavi SD, et al. An Approach to a Rare Case of Collision Tumor of Follicular and Papillary Carcinomas of the Thyroid. Int J Otorhinolaryngol Clin 2020;12(2):35-37.

Source of support: Nil

Conflict of interest: None
}

pattern. The neoplasm showed capsular and vascular invasion and was suggestive of a minimally invasive follicular carcinoma of the right lobe of thyroid (Figs 1 and 2).

Subsequently, considering the size of the tumor and associated risk factors, a completion thyroidectomy with central compartment clearance was planned. Intraoperatively, the left lobe of thyroid was found to be normal in size and vascularity. There were no enlarged nodes in the central compartment. Surgery went off smoothly without any complications. Histopathologically, the specimen was reported as a papillary carcinoma of the left lobe of thyroid measuring $0.7 \mathrm{~cm} \times 0.4 \mathrm{~cm} \times 0.2 \mathrm{~cm}$ with associated thyroiditis. Lymph node biopsy showed no evidence of metastasis.

Whole body iodine scan done two months later showed a single focus of increased uptake in the region of the thyroid bed with no distant metastasis. Radioactive iodine ablation was accordingly done with I-131. The patient was later started on suppressive doses of L-thyroxine. He is presently asymptomatic for almost a year and has been advised regular follow-up and whole body iodine scan every year for at least up to three years (Fig. 3). 


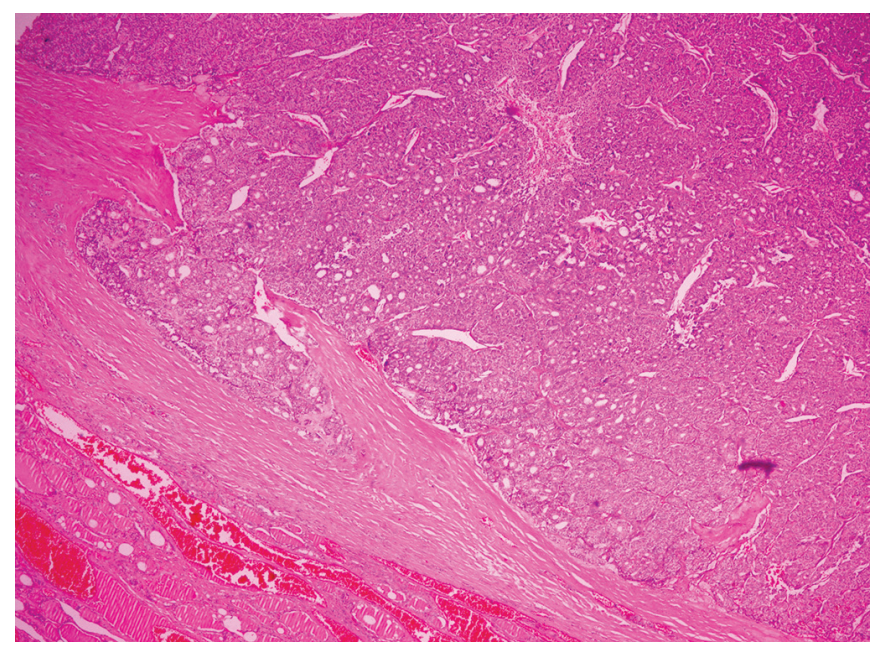

Fig. 1: Microscopic picture of follicular carcinoma of the right lobe of thyroid showing vascular and capsular invasion on $\mathrm{H}$ and $\mathrm{E}$ stains

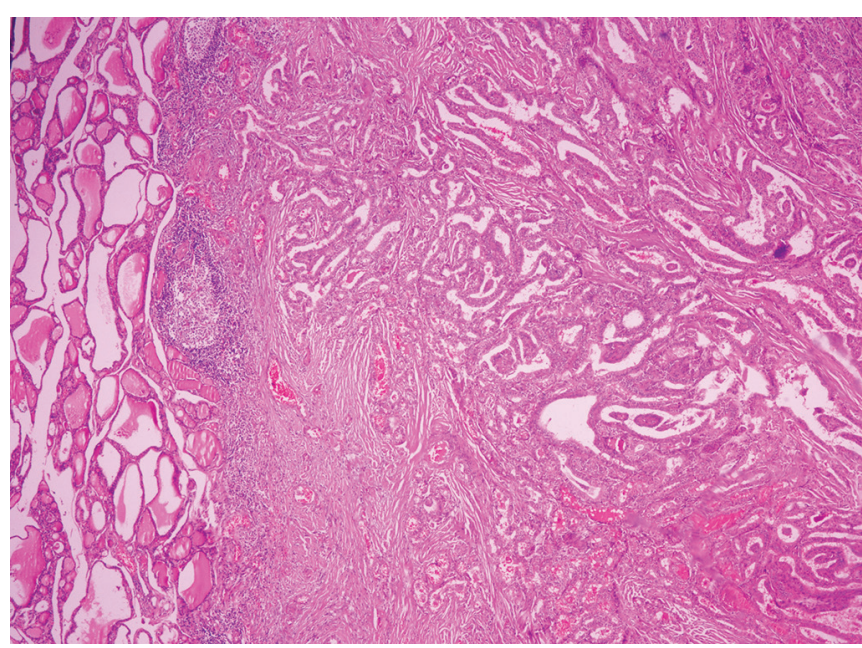

Fig. 2: Microscopic picture of papillary carcinoma of the left lobe of thyroid with associated thyroiditis on $\mathrm{H}$ and $\mathrm{E}$ stains
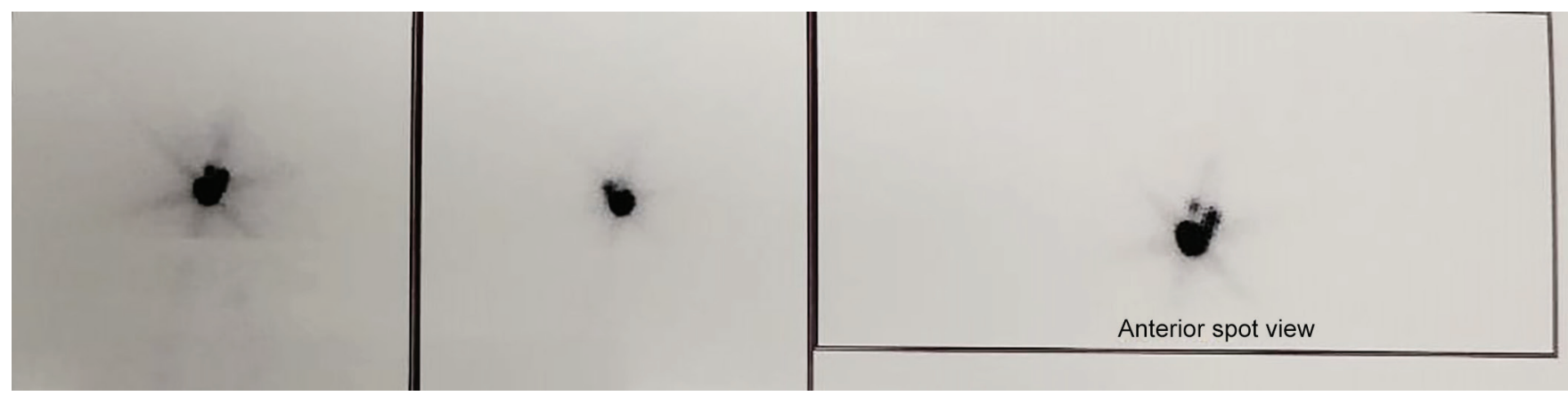

Fig. 3: Whole body iodine scintigraphy showing residual thyroid tissue in the thyroid bed with no distant metastasis

\section{Discussion}

Thyroid malignancies constitute the most common type of endocrine cancer. Of all the thyroid malignancies, papillary carcinoma was encountered to be the commonest and accounted for $89.8 \%$ of all thyroid malignancies. ${ }^{7}$ The main histologic types of thyroid carcinoma are (1) differentiated thyroid carcinoma (including papillary, follicular, and Hurthle cell carcinoma); (2) medullary thyroid carcinoma; and (3) anaplastic thyroid carcinoma. ${ }^{8}$ Solitary tumors of the thyroid are common, but it is rare for the gland to harbor two distinct types of malignancies at the same time.

Multiple challenges may be encountered while dealing with a follicular neoplasm of undetermined significance. FNAC cannot distinguish between a follicular adenoma, follicular carcinoma, and follicular variant of papillary carcinoma. ${ }^{9}$ Further, follicular neoplasm cannot be defined accurately on frozen section assessment either; hence, the role of frozen section in the diagnosis of follicular carcinoma intraoperatively is minimal. ${ }^{10}$ Only a paraffin section of the excised specimen showing vascular and/or capsular invasion can confirm the diagnosis of a follicular carcinoma. $^{11}$

Hence in our case, even at the time of completion thyroidectomy, frozen section assessment was not done considering it was a follicular carcinoma on the right side. Also, considering the size of the tumor which was more than four centimeters, a clearance of the central compartment of the neck lymph nodes was performed. ${ }^{12}$ However in spite of a completely normal and non-suspicious appearance at the time of surgery, the left thyroid lobe was reported as having a papillary carcinoma of thyroid with thyroiditis. No metastasis was reported in the central compartment nodes.

Although both follicular and papillary carcinomas carry a good prognosis, they have the potential to spread hematogenously and by lymphatic means, respectively. ${ }^{13}$ This scenario increases the potential risk of lymphovascular metastasis, and hence, the surgeon should bear in mind the possibility of the same.

Whole body iodine scan with I-131 done 2 months after the completion thyroidectomy showed traces of residual thyroid tissue in the neck and no distant metastasis, thus requiring the need for radioactive I-131 ablation. Repeat whole body iodine scans are advisable in all cases annually for a period of at least three years post-surgery.

To summarize, collision tumors of the thyroid are rare entities as such, while the coexistence of papillary and follicular carcinomas together is even rarer. One or even both components of the tumor may be silent and slow-growing, and the patient may be completely asymptomatic for a significant amount of time in spite of harboring 
two histologically distinct primary tumors in the thyroid. The site of the two tumors may be in the same lobe or two separate lobes as was seen in this case.

Usually, one of the two tumors will increase in size earlier than the other and produce symptoms, which may or may not indicate a malignancy. Thorough clinical examination and a complete array of investigations may not be sufficient to establish a diagnosis, especially in a case of follicular neoplasm. However, they are extremely useful in diagnosing a papillary or medullary carcinoma or any other benign thyroid lesion and hence must be performed in every case of a palpable thyroid swelling.

Treatment options will vary depending upon the diagnosis of the primary tumor. Frozen section is only useful in case the primary tumor is a papillary carcinoma and may provide the road map for a completion thyroidectomy and/or lymphatic clearance at the same setting. However, it may not provide the adequate details as regards the diagnosis of a follicular carcinoma. Only paraffin sections can diagnose the latter and only then can further treatment be planned.

Size of the tumor, risk factors, and the histological type of the primary malignancy will decide the further management of these patients at this stage. ${ }^{12}$ All management options including a completion thyroidectomy, central and lateral lymphatic clearance, I-131 ablation therapy, and radiotherapy must be kept in mind, and the surgeon must follow the established protocol according to the type of the primary tumor.

Histological type of the second tumor may further complicate things, and it is important to state here that the surgeon may be completely unaware of a second malignancy at this stage, especially if it presents with no symptoms and is reported as a normal-looking thyroid gland on investigations. This headache to the primary surgeon may be prevented if the second malignancy gets presented in the same thyroid lobe as the first, which was unfortunately not the case in our patient. So, diagnosis of a second primary malignancy of the thyroid can occur as a surprise to the surgeon but more importantly a big shock to the patient, who may already be under a considerable amount of stress due to multiple investigations, surgery, and knowledge of primary malignancy.

It is therefore extremely important to counsel the patient thoroughly before performing any kind of secondary procedure, once the diagnosis of primary malignancy is made. This counseling should include a complete explanation of the chances of a second primary tumor, however meager they may be. Once the diagnosis of both the tumors is established, a whole body iodine scan is mandatory and further management will depend on the histological types of both the tumors and nuclear medicine reports. Here again, the patient should be counseled well as diagnosis of multiple malignancies can be confusing as well as demoralizing, while the head and neck surgery team should always keep their colleagues in endocrinology, nuclear medicine, and radiotherapy in the loop and seek their help and advice whenever necessary.

Finally, good follow-up and repeated scanning are essential parts in successful management of collision tumors of the thyroid, just like any other primary thyroid malignancy.

\section{Conclusion}

The idea behind presenting this case is to make the clinician aware of the possibility of a collision tumor when dealing with a thyroid malignancy. Also, it is important to note that preoperative investigations may not be entirely reliable in a thyroid malignancy, even less so in cases of collision tumors. Finally, as there are no recommended protocols, the clinician must be flexible, keeping in mind all kinds of treatment options, always keeping the patient in the loop, while managing a case of collision tumor of the thyroid.

\section{References}

1. Brandwein-Gensler M, Urken M, Wang B. Collision tumor of the thyroid: a case report of metastatic liposarcoma plus papillary thyroid carcinoma. Head Neck 2004;26(7):637-641. DOI: 10.1002/hed.20024.

2. Zhang Z, Min J, Yu D, et al. Renal collision tumour of papillary cell carcinoma and chromophobe cell carcinoma with sarcomatoid transformation: a case report and review of the literature. Can Urol Assoc J 2014;8(7-8):E536-E539. DOI: 10.5489/cuaj.1811.

3. Ryan N, Walkden G, Lazic D, et al. Collision tumors of the thyroid: a case report and review of the literature. Head Neck 2015;37(10):E125-E129. DOI: $10.1002 /$ hed.23936.

4. Plauche V, Dewenter T, Walvekar RR. Follicular and papillary carcinoma: a thyroid collision tumor. Indian J Otolaryngol Head Neck Surg. 2013;65(Suppl 1):182-184. DOI: 10.1007/s12070-011-0450-0.

5. Thomas VP, George R. Collision tumors of the thyroid: review of literature and report of a case of Papillary-Follicular collision tumor. Thyroid Res Pract 2018;15:60-64.

6. Cracolici V, Mujacic I, Kadri S, et al. Synchronous and metastatic papillary and follicular thyroid carcinomas with unique molecular signatures. Endocr Pathol 2018;29(1):9-14. DOI: 10.1007/s12022-0179491-6.

7. Noone AM, Howlader N, Krapcho M, et al., eds. SEER Cancer Statistics Review, 1975-2015, based on November 2017 SEER data submission, posted to the SEER web site, April 2018. Bethesda, MD: National Cancer Institute; 2018. Available at: https://seer.cancer.gov/ csr/1975_2015/.

8. Haddad R, Nasr C, Bischoff $L$, et al. NCCN guidelines insights: thyroid carcinoma, version 2.2018. J Natl Compr Canc Netw 2018;16(12):14291440. DOI:10.6004/jnccn.2018.0089.

9. Moorthy R, Kumar S, Warfield A. Thyroid and parathyroid gland pathology. In: Watkinson J, Clarke R, editors. Scott-Brown's Otorhinolaryngology Head and Neck Surgery. 8th ed. Vol. 1. Boca Raton: CRC Press; 2018. p. 654.

10. Moorthy R, Kumar S, Warfield A. Thyroid and parathyroid gland pathology. In: Watkinson J, Clarke R, editors. Scott-Brown's Otorhinolaryngology Head and Neck Surgery. 8th ed. Vol. 1. Boca Raton: CRC Press; 2018. p. 687.

11. Maitra A. The endocrine system. In: Kumar V, Abbas A, Aster J, editors. Robbins \& Cotran Pathologic Basis of Disease. 9th ed. Philadelphia: Elsevier; 2014. p. 1100.

12. Mehanna H, Boelart K, Sharma N. Management of differentiated thyroid cancer. In: Watkinson J, Clarke R, editors. Scott-Brown's Otorhinolaryngology Head and Neck Surgery. 8th ed. Vol. 1. Boca Raton: CRC Press; 2018. p. 753.

13. Moorthy R, Kumar S, Warfield A. Thyroid and parathyroid gland pathology. In: Watkinson J, Clarke R, editors. Scott-Brown's Otorhinolaryngology Head and Neck Surgery. 8th ed. Vol. 1. Boca Raton: CRC Press; 2018. p. 667-670. 